\title{
ANÁLISE TÉRMICA DO PROCESSO DE INCINERAÇÃO DE RESÍDUOS AQUOSOS COM CHAMAS ENRIQUECIDAS
}

\author{
Pedro Teixeira Lacava ${ }^{1}$ \\ João Andrade de Carvalho $\mathrm{Jr}^{2}$ \\ Amilcar Porto Pimenta ${ }^{3}$ \\ Marco Aurélio Ferreira ${ }^{4}$
}

Resumo. A utilização de oxigênio para enriquecer o comburente pode ser uma :ecnica atrativa para aumentar a capacidade de incineração de uma câmara de combustão projetada para operar com ar. Para uma determinada vazão de combustivel. se alguns parâmetros forem fixados (temperatura de operação, nível de turbulência e tempo de residência), ao se adicionar oxigênio no ar de combustão é possivel aumentar a capacidade de incineração da câmara. Desta forma, o presente trabalho apresenta uma análise térmica do enriquecimento do oxidante em uma câmara de escala laboratorial projetada para incineração de resíduos líquidos aquosos, utilizando diesel como combustivel auxiliar. Os resultados experimentais indicam que a análise térmica pode ser uma importante ferramenta para previsão do aumento da capacidade de incineração da câmara.

Palavras-chave: incineração, combustão com enriquecimento do oxidante, análise térmica.

58 Congresso Anual da ABM, Julho 2003, Rio de Janeiro.

1 - Professor Adjunto/ Instituto Tecnológico de Aeronáutica - São José dos Campos

2 - Professor Titular/ Universidade Estadual Paulista - Guaratinguetá

3 - Professor Adjunto/ Instituto Tecnológico de Aeronáutica - São José dos Campos

4 - Pesquisador/ Instituto Nacional de Pesquisas Espaciais - Cachoeira Paulista 


\section{INTRODUÇÃO}

A grande maioria dos processos de combustão utilizam o ar ambiente como fonte de oxigênio. No entanto, a maior parte da energia térmica liberada é absorvida pelo nitrogênio, visto que o ar em base volumétrica é constituído de $78 \%$ de nitrogênio, $21 \%$ de oxigênio e $1 \%$ de traços de outros constituintes. Do ponto de vista térmico, o nitrogênio é inerte, ou seja, apenas absorve a energia liberada pelas reações de combustão, que poderia estar sendo utilizada em um processo industrial (Griffith, 1990). Desta forma, enriquecer o oxidante nada mais é que aumentar a sua porcentagem de $\mathrm{O}_{2}$ acima dos $21 \%$ encontrados no ar atmosférico, podendo atingir os seguintes benefícios (Flamme e Kremer, 1993): aumento da produtividade, elevação da eficiência térmica, redução do volume dos gases de combustão, aumento da temperatura de operação e redução do consumo de combustivel. $O$ uso de oxigênio para enriquecer o oxidante pode ser uma alternativa atrativa para aumentar a capacidade de incineração de uma câmara projetada para operar com ar como oxidante, principalmente para residuos de baixo poder calorifico (Sharani et al., 1996). Para uma certa vazão de combustivel, se alguns parâmetros de incineração forem mantidos constantes (como temperatura da câmara, nivel de turbulência e tempo de residência), o aumento da taxa de incineração torna-se possivel com a injeção de oxigênio (Melo et al., 1998). Desta forma, o presente trabalho apresenta uma investigação teórica e experimental sobre o aumento da capacidade de incineração através do enriquecimento do ar, para uma câmara projetada para incineração de resíduos aquosos e que utiliza o diesel como combustivel auxiliar e o ar como oxidante.

\section{ANÁLISE TERMOQUÍMICA}

Nesta seção realizou-se uma abordagem teórica baseada nos balanços de massa e energia aplicados à situação de incineração de resíduos aquosos com combustão enriquecida. Através da análise termoquímica é possível estimar a capacidade do aumento da taxa de incineração da câmara com o enriquecimento. $O$ combustivel utilizado na análise foi o diesel (aproximado como $\mathrm{C}_{12} \mathrm{H}_{26}$ ) e o residuo considerado como $100 \%$ água. Essa situação teórica corresponde à condição experimental investigada nas seções seguintes, permitindo a comparação.

Os balanços de massa e de energia foram aplicados ao volume de controle (VC) definido como sendo o volume de toda câmara de combustão.

Adotou-se como condição de entrada os reagentes e o resíduo estando a $25^{\circ} \mathrm{C}$ e $1 \mathrm{~atm}$. Como condição de saída os gases deixam o VC na temperatura $T_{\mathrm{s}}$ e a $1 \mathrm{~atm}$. Também foi incluída ao balanço de energia a eficiência da refratagem da câmara, ou seja, o calor que é transferido para parede. Além disso, algumas considerações foram feitas: a) combustão completa, ou seja, não há dissociação dos produtos de combustão; b) a água formada decorrente da oxidação do hidrogênio presente no combustivel está em fase gasosa; c) o resíduo é totalmente vaporizado; d) a combustão ocorre a pressão constante.

\subsection{Balanço de Massa}

A equação (1) apresenta a reação global de combustão com excesso normalizado de ar enriquecido e injeção de residuo. 


$$
\begin{aligned}
\mathrm{C}_{12} \mathrm{H}_{26} & +b \cdot 18,5 \cdot \mathrm{O}_{2}+\text { b. }(18,5-a) \cdot 3,76 \cdot \mathrm{N}_{2}+c \cdot \mathrm{H}_{2} \mathrm{O}_{(R E S)} \rightarrow \\
& \rightarrow 12 \cdot \mathrm{CO}_{2}+13 \cdot \mathrm{H}_{2} \mathrm{O}+\text { b. }(18,5-a) \cdot 3,76 \cdot \mathrm{N}_{2}+18,5 \cdot(b-1) \cdot \mathrm{O}_{2}+c \cdot \mathrm{H}_{2} \mathrm{O}_{(R E S)},
\end{aligned}
$$

onde a é número de moles de oxigênio não proveniente do ar oxidante, se $a=0$ o oxidante é $100 \%$ ar e se $a=18,5$ o oxidante é $100 \%$ oxigênio; $\boldsymbol{b}$ é o excesso de ar genérico normalizado; $c$ é o número de moles de resíduo aquoso acrescentado.

\subsection{Balanço de Energia}

Conforme já apontado, o processo de combustão foi considerado como sendo a pressão constante. Assim, a primeira lei da termodinâmica aplicada ao VC resulta no seguinte balanço de energia:

$H_{R}=H_{P}+\Delta H_{R E S}+Q_{\text {parede }}$,

onde $H_{R}$ é a entalpia dos reagentes na entrada do VC, $H_{p}$ a entalpia dos produtos de combustão na saida do $V C, \Delta H_{R E S}$ a energia transferida para o resíduo e $Q_{\text {parede }} O$ calor transferido à parede da câmara. Escrevendo a equação (2) em termos de calores especificos, poder calorífico inferior do combustivel e considerando que os reagentes entram na câmara a $25^{\circ} \mathrm{C}$ e $1 \mathrm{~atm}$, temos:

$n_{C 12 H 26} \cdot P C l_{C 12 H 26}=\int_{25}^{T s}\left(\sum_{j} n_{j} \cdot C p_{j}\right) \cdot d T+n_{H 2 O / R E S} \cdot\left(\int_{25}^{T s} C p_{H 2 O / v a p o r} \cdot d T+h_{L}\right)+Q_{\text {parede }}$,

onde $n_{\mathrm{C} 12 \mathrm{H} 26}$ é o número de moles do combustível, $P C l$ é o poder calorífico inferior do combustível, $n_{j}$ é o número de mols para o composto $j$ dos gases produtos de combustão $\left(j=\mathrm{CO}_{2}, \mathrm{H}_{2} \mathrm{O}, \mathrm{O}_{2}\right.$ e $\left.N_{2}\right), n_{\text {H2O/RES }}$ é o número de moles da água que simula o resíduo, $h_{L}$ o calor latente de vaporização da água e Cp o calor específico a pressão constante. Desta forma, a avaliação do processo de enriquecimento do ponto de vista termoquímico, consiste do acoplamento das equações (1) e (3). 0 $P C l$ do $\mathrm{C}_{12} \mathrm{H}_{26}$ é $10259 \mathrm{kcal} / \mathrm{kg}$ ou $1744.10^{3} \mathrm{cal} / \mathrm{mol}$ e o $h_{L}$ da água igual $-539 \mathrm{kcal} / \mathrm{kg}$ ou $-9720 \mathrm{cal} / \mathrm{mol}$. Os valores de Cp foram obtidos em Perry e Chilton (1973).

\subsection{Simulação}

Nesta subseção são apresentados os resultados das simulações de condições de enriquecimento em uma câmara inicialmente projetada para operar com ar. Para avaliar o efeito do enriquecimento no aumento da capacidade de incineração se utilizou como indicador a taxa de incineração, definida como a razão entra a vazão mássica de resíduo ( $\left.m_{\mathrm{res}}\right)$ e a vazão mássica de combustível $\left(m_{\mathrm{f}}\right)$.

Figura 1 apresenta o resultado do comportamento da taxa de incineração de uma câmara de incineração de residuo aquoso (água), queimando diesel $\left(\mathrm{C}_{12} \mathrm{H}_{26}\right)$ como combustivel auxiliar e com eficiência da câmara constante em $80 \%$ para os vários níveis de enriquecimento (\% de $\mathrm{O}_{2}$ no oxidante) e excesso de oxidante normalizado. Como parâmetro fixo de operação estipulou-se como temperatura na saida da câmara $\left(T_{s}\right) 1030 \mathrm{~K}$ (valor adotado experimentalmente, conforme descrito na seção seguinte).

Nota-se que para qualquer valor de $\boldsymbol{b}$ há um aumento considerável na taxa de incineração até aproximadamente $50 \%$ de $\mathrm{O}_{2}$ no oxidante. As curvas ainda mostram que há uma maior eficiência para excessos de oxidante mais elevados. Por exemplo: para $b=1,15$, passando de $21 \%$ para $100 \%$ de $\mathrm{O}_{2}$ presente no oxidante, a 
taxa de incineração aumento $51 \%$. Já para $b=2,00$ a aumento é de $172 \%$. Ambos comportamentos estão atrelados às combinações de vazão na câmara. A Figura 2 apresenta as vazões mássicas total, do residuo, do $\mathrm{O}_{2}$ injetado e do ar, para a vazão de combustivel de $3,11 \mathrm{~g} / \mathrm{s}(133,55 \mathrm{~kW})$.

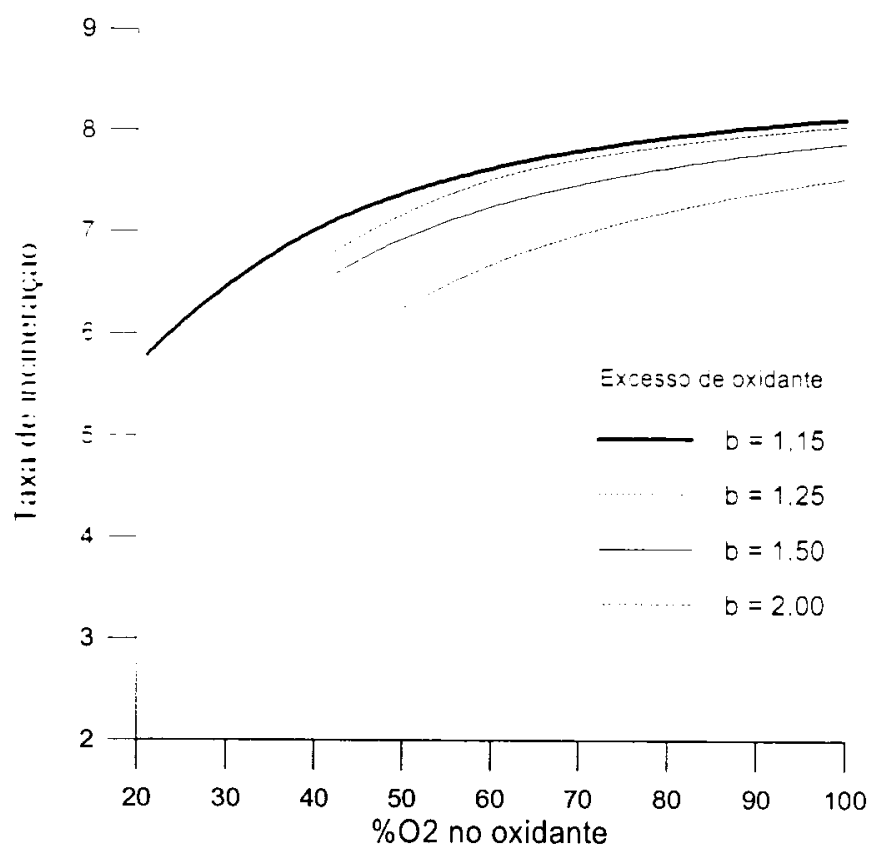

Figura 1. Comportamento da taxa de incineração em função do $\mathrm{O}_{2}$ no oxidante para diversos valores de excesso de oxidante normalizado (eficiência da câmara é $80 \%$ ).

Como mostram as curvas da Figura 2, a redução de ar na câmara com o enriquecimento é bastante acentuada até aproximadamente $50 \%$ de $\mathrm{O}_{2}$ no oxidante. Deste ponto em diante, a redução ocorre de maneira mais branda. Como a vazão total de oxigênio $\left(\mathrm{O}_{2}\right.$ do $\mathrm{ar}+\mathrm{O}_{2}$ injetado) é constante para cada valor de excesso de oxidante, basicamente o responsável pelo comportamento de elevado aumento da taxa de incineração até $50 \%$ de $\mathrm{O}_{2}$ é a acentuada redução da quantidade de nitrogênio até esse ponto.

Isso também explica o fato da diferença entre as taxas de incineração para cada valor de excesso de oxidante irem diminuindo à medida que se aumenta o teor de oxigênio no oxidante. Para elevados niveis de enriquecimento, onde a diferença entre as vazões de ar para cada valor de excesso de oxidante é pequena, o que acaba sendo mais importante no valor da taxa de incineração é o oxigênio excedente injetado na câmara.

\section{ABORDAGEM EXPERIMETAL}

\subsection{Descrição do Experimento}

Em todos ensaios realizados, utilizou-se apenas água para simulação do residuo, em virtude das facilidades experimentais envolvidas e pelo fato do enriquecimento do oxidante ser vantajoso para incineração de residuos não combustiveis. Além disso, o presente trabalho tem como objetivo caracterizar o balanço de energia na câmara e não a destruição térmica de compostos presentes no resíduo. 

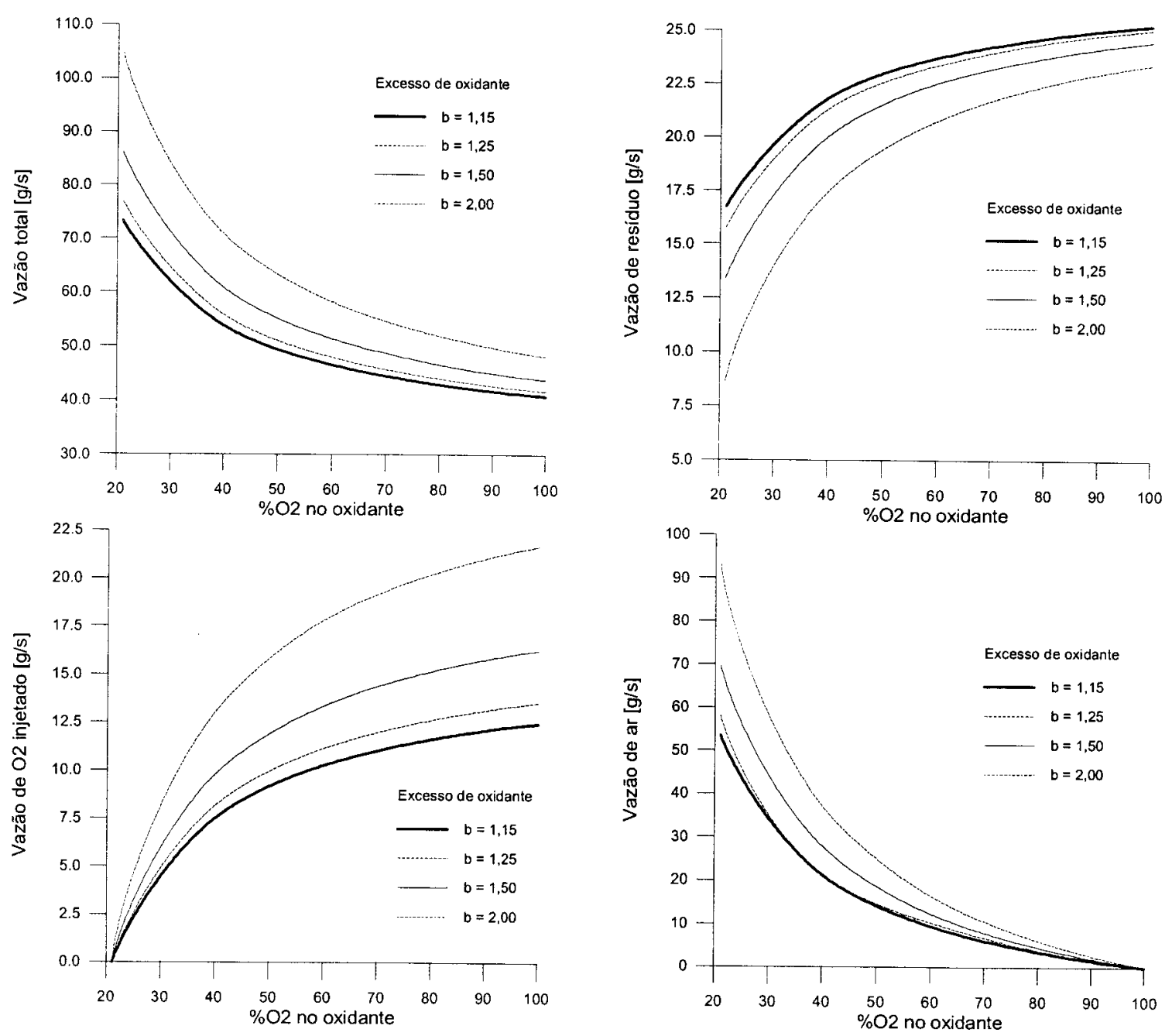

Figura 2. Vazões em função da porcentagem de $\mathrm{O}_{2}$ no oxidante e do seu excesso (b), para a vazão de combustivel de $3,11 \mathrm{~g} / \mathrm{s}$ e eficiência de $80 \%$ para câmara.

Os ensaios foram realizados em uma câmara de combustão vertical, com parede refratária de $0,15 \mathrm{~m}$ de espessura, sem refrigeração externa e com o volume total de 1000 litros. Como combustível auxiliar utilizou-se diesel, injetado na câmara através de um queimador com dispositivo para ancorar a chama do tipo swirler. A Figura 3 apresenta um esquema da câmara, com o queimador posicionado em sua extremidade inferior. A injeção de resíduo é feita por um injetor tipo $Y$-jet, de tal forma que o spray formado não incida completamente sobre a chama para não apagá-la.

A instrumentação associada à câmara conta com medidas de temperatura por termopares nas posições: $0,18,0,70,1,05,1,40,1,75,2,50,3,00$ e 4,20 m a jusante do queimador; análise dos gases de combustão $\left(\mathrm{CO}, \mathrm{CO}_{2}, \mathrm{O}_{2}\right.$ e $\left.\mathrm{NO}_{\mathrm{x}}\right)$ através de amostragens continuas feitas por duas sondas devidamente refrigeradas, posicionadas em dois pontos da câmara: na região próxima ao final da chama, correspondendo ao volume 227 litros, e a $30 \mathrm{~cm}$ da saída da câmara, correspondendo ao volume de 997 litros; medidas das vazões de diesel, resíduo, ar e oxigênio, feitas através de rotâmetros. 


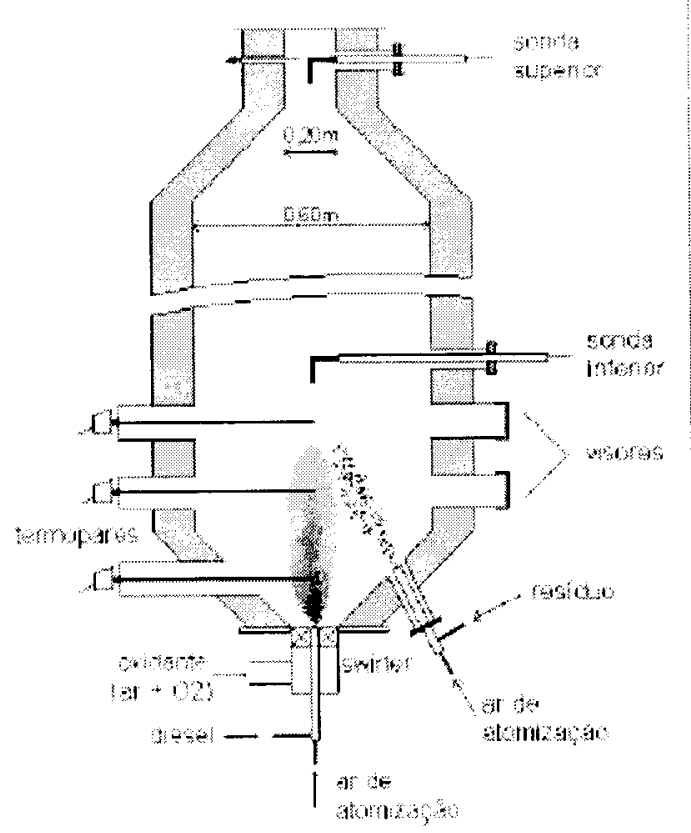

Figura 4. Esquema da câmara de combustão.

Para os ensaios, manteve-se a vazão de diesel constante em $3,11 \mathrm{~g} / \mathrm{s}$, o que representa uma potência de 133,55 kW. As vazões de ar e de oxigênio foram combinadas de tal forma que a vazão mássica do oxigênio na saída da câmara fosse $1,58 \mathrm{~g} / \mathrm{s}$. Desta forma, a queima sempre ocorreu com excesso de oxigênio em torno de $15 \%$. Finalmente, o aproveitamento da energia disponibilizada pela redução da presença de nitrogênio foi feito ajustando a vazão de resíduo, mas mantendo a temperatura na saída da câmara em $1030 \mathrm{~K}$. A injeção de oxigênio puro foi feita na linha de ar oxidante antes da passagem do mesmo pelo queimador.

\subsection{Resultados e Discussões}

A Figura 4(a) apresenta o comportamento experimental da taxa de incineração, definida como a razão entre as vazões mássica de resíduo e combustivel (mres/mf), em função da porcentagem de oxigênio no oxidante. Percebe-se que há um aumento considerável até aproximadamente o nível de enriquecimento de $50 \%$ de oxigênio, cerca de $85 \%$ acima da operação com ar. No entanto, a partir deste ponto, a taxa de incineração aumenta "Ientamente" com o incremento da porcentagem de $\mathrm{O}_{2}$ no oxidante. Para combustão com oxi-chama $\left(100 \% \mathrm{O}_{2}\right)$, o aumento da taxa de incineração foi de $111 \%$ em relação à operação com ar e $13,4 \%$ em relação ao enriquecimento de $50 \%$ de $\mathrm{O}_{2}$. As razões para o comportamento mais positivo para até $50 \%$ de oxigênio são as mesmas discutidas na seção anterior durante a análise termoquímica. A Figura 4(b) mostra que os comportamentos das vazões obtidas experimentalmente para a quantidade resíduo injetado, ar, oxigênio e total, seguem os mesmos padrões apresentados na abordagem teórica.

Além dos resultados experimentais, a Figura 4(a) também apresenta da taxa de incineração com enriquecimento calculado a partir do modelo termoquímico apresentado na seção 2. Contudo, para aplicação do modelo é preciso estimar a eficiência da refratagem da câmara, ou seja, a quantidade de calor transferida para parede da mesma. Na prática, em uma instalação de incineração de residuos 
líquidos aquosos, onde se deseja adaptar um sistema de enriquecimento, o único dado de transferência de calor possivel de ser obtido é o da condição atual de operação da instalação, ou seja, para o oxidante sendo ar. Assim, o resultado calculado na Figura 4(a) foi obtido desta forma. A partir dos dados experimentais para operação com ar (análise de gases, vazões e temperatura na saída da câmara) utilizou-se a equação (3) para calcular a quantidade de calor transferida para parede da câmara. Com isto, encontrou-se que $39,17 \%$ da energia liberada pela combustão é perdida para parede da câmara e tal valor foi adotado constante para o cálculo da taxa de incineração com o enriquecimento. Utilizando tal procedimento, encontramos uma diferença média de $9,4 \%$ entre os resultados experimentais e os calculados (máxima de $12,59 \%$ e mínima de $6,12 \%$ para $100 \%$ e $24,98 \%$ de $\mathrm{O}_{2}$, respectivamente).

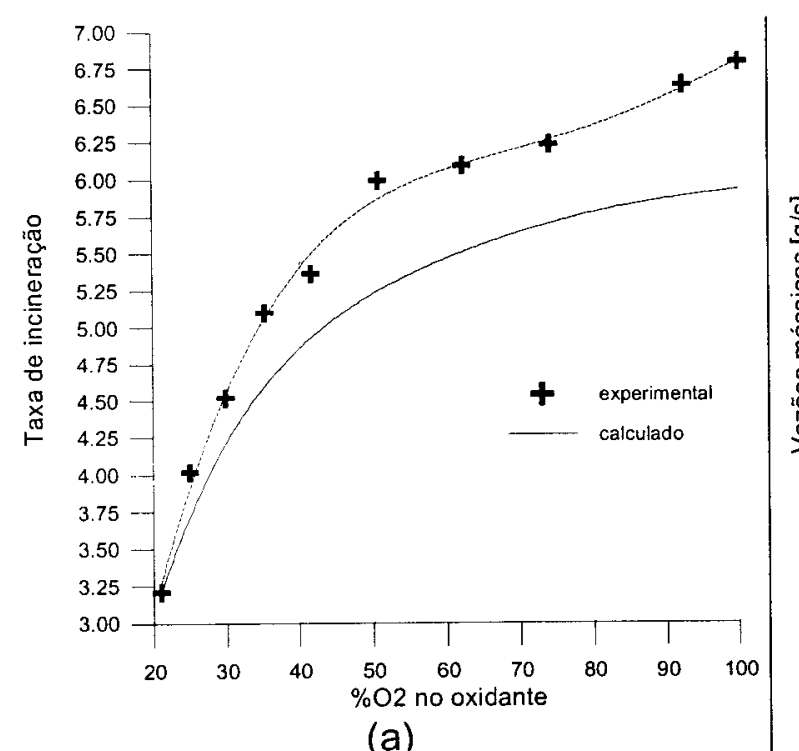

(a)

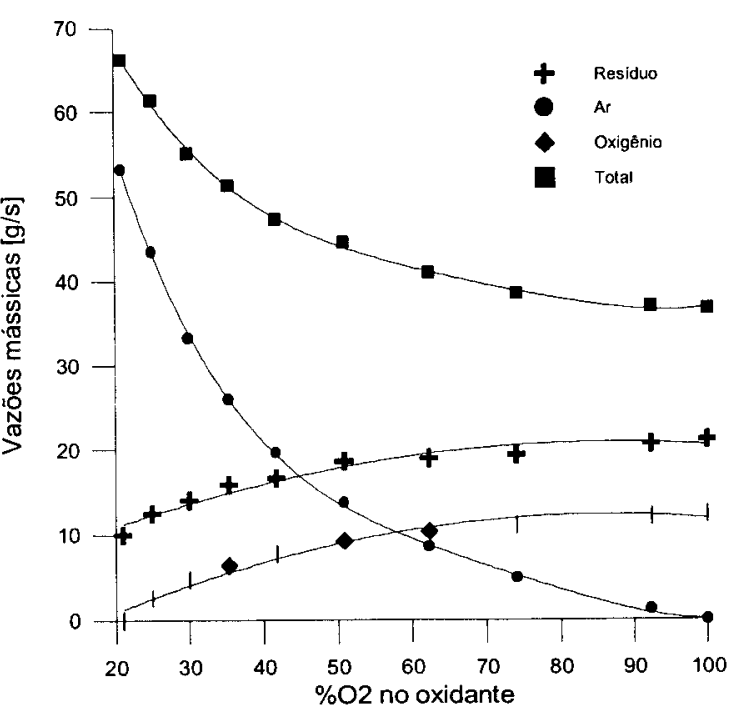

(b)

Figura 4 - (a) - Resultados experimentais e calculados sobre o comportamento da taxa de incineração em função da porcentagem de $\mathrm{O}_{2}$ no oxidante. (b) Vazões de resíduo, ar, oxigênio e total, em função da $\% \mathrm{O}_{2}$ no oxidante.

A Figura 4(a) sugere que o cálculo considerando que energia perdida para parede é constante e igual a da operação com ar, subestima a capacidade do aumento da taxa de incineração com enriquecimento. A energia liberada pelas reações de combustão em processos de incineração segue basicamente três caminhos diferentes: 1) eliminada com os gases produtos de combustão, 2) transferida para carga de resíduo e 3) transferida para as paredes da câmara. Quando a queima ocorre sobre condições enriquecidas, a energia eliminada pelos produtos de combustão diminui, pois a massa total de gases é reduzida se a temperatura for mantida constante. Sendo assim, essa energia disponibilizada seguirá os outros dois caminhos.

Para analisar esse contexto sobre os resultados experimentais, realizou-se um balanço de energia na câmara de combustão, aplicando o equacionamento apresentado na seção 2 com os resultados obtidos experimentalmente para as vazões, concentrações e temperaturas. Além disso, considerou-se combustão completa, em função das emissões de $\mathrm{CO}$ e $\mathrm{NO}_{x}$ serem muitos baixas na saída da 
câmara, quando comparadas com as do $\mathrm{CO}_{2}, \mathrm{O}_{2}, \mathrm{~N}_{2}$ e $\mathrm{H}_{2} \mathrm{O}$. O resultado está apresentado na Figura 5.

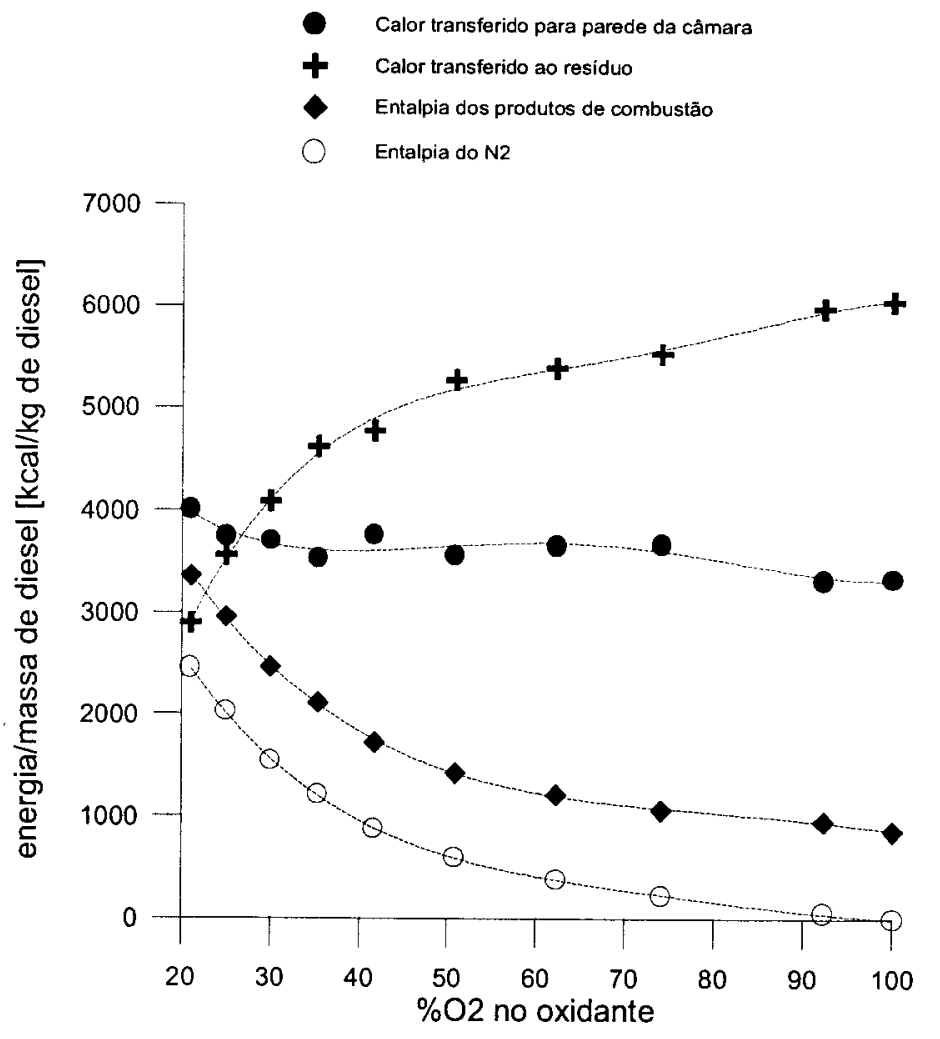

Figura 5. Resultado do balanço de energia da câmara.

O balanço de energia mostra que há uma redução na razão calor total transferido para parede da câmara/massa de combustível e que toda energia disponibilizada pela redução da massa de nitrogênio no oxidante é aproveitada para aumentar a vazão de resíduo. A quantidade de calor transferida da chama e dos gases de combustão para parede da câmara basicamente ocorre por convecção e radiação, além da influência do tempo de troca térmica.

Conforme apresentado na Figura 4(b), com aumento do enriquecimento há uma redução na massa total de gases que escoa pela câmara. Como a distribuição de temperatura altera-se muito pouco após a injeção de resíduo, de acordo com os resultados dos termopares instalados ao longo da câmara; o principal fator que acaba modificando a taxa de transferência de calor convectiva para parede da câmara, à medida que se aumenta o nível de enriquecimento, è a diminuição de velocidade em decorrência da menor vazão mássica de gases.

Para a transferência de calor por radiação, o raciocinio já não é tão simples como no caso da convecção. Diversos fatores, dos quais depende a sua intensidade, são fortemente afetados pelo processo de enriquecimento e de maneira desigual, alguns favorecendo e outros reduzindo sua taxa de transferência por radiação. Como modificações que reduzem a troca térmica por radiação pode-se citar: 1) menor presença de fuligem nos gases, 2) menor presença de compostos heteropolares $\left(\mathrm{CO}_{2}\right.$ e $\left.\mathrm{H}_{2} \mathrm{O}\right)$ na região de chama em razão da dissociação como conseqüência do aumento de temperatura; 3) redução do comprimento característico da chama diminuindo sua emissividade. No lado do aumento da troca 
térmica por radiação tem-se: 1) aumento de temperatura na região de chama; 2) recombinação de compostos decorrentes da dissociação para formação de moléculas heteropolares $\left(\mathrm{CO}_{2}\right.$ e $\left.\mathrm{H}_{2} \mathrm{O}\right)$ na região de pós chama; 3 ) elevada presença de vapor d'água após a injeção de residuo. Os dois últimos itens citados produzem pouca influência em razão de serem regiões de menor temperatura. De qualquer forma, quantificar o efeito do enriquecimento nesse processo é uma tarefa complexa; no entanto, acredita-se na média há uma redução na transferência de calor por radiação para parede da câmara.

Outro ponto importante é o tempo de residência dos gases na câmara, ou seja, o tempo de troca térmica entre os gases e a parede. Como a vazão total diminui com enriquecimento, o tempo de residência aumenta e, como conseqüência, a quantidade total de calor transferido também aumentará. O tempo de residência calculado para operação com ar e oxigênio puro foi de 4,2 e 6,5 segundos, respectivamente. No entanto, esse aumento no tempo de residência não compensa a redução nas taxas de transferência de calor por radiação e convecção.

\section{4 - CONCLUSÕES}

1) Os cálculos mostraram que há um considerável aumento da capacidade de incineração até $50 \%$ de $\mathrm{O}_{2}$ no oxidante, o que foi confirmado experimentalmente. A análise teórica ainda mostrou que a eficiência do enriquecimento aumenta com o também aumento do excesso de oxidante.

2) O comportamento da taxa de incineração foi previsto teoricamente e observado experimentalmente, Contudo, quando o modelo é aplicado considerando a taxa de transferência de calor constante e igual a obtida experimentalmente para operação com ar, menores taxas de incineração são observadas em comparação com as obtidas experimentalmente, em qualquer nível de enriquecimento. A diferença média foi de $9,4 \%$.

3) Do ponto de vista de eficiência energética, duas importantes conclusões podem ser extraidas para presente aplicação. A primeira é que toda energia disponibilizada da redução da quantidade de nitrogênio no oxidante é completamente utilizada para aumentar a capacidade de incineração. A segunda é que, já que taxa de transferência de calor através da parede reduz com enriquecimento, mais energia estará disponível para aumentar a capacidade de incineração.

\section{5 - REFERÊNCIAS}

Flamme, M.; Kremer, H., 1993, "Reduction of the $\mathrm{NO}_{x}$ Output From Industrial Burners Firing a Mixture of Fuel Gas and Combustion Air Enriched and Oxygen", Proceedings of the Second International Conference on Combustion Technologies for a Clean Environment, Lisbon, Portugal, p.p. 1-7.

Melo, G.F.; Lacava, P.T.; Carvalho, J.A.Jr., 1998, "A Case Study of Air Enrichment in Rotary Kiln Incineration", International Communications in Heat and Mass Transfer, 25(5):681-692.

Griffith, C.R.,1990, "Diverse Experience Using Oxygen Systems in Waste Incineration", presented at the $4^{\text {th }}$ Annual National Symposium on Incineration of Industrial Wastes, Houston, Texas

Shahani, G.H.; Gunardson, H.H.; Easterbrook, N.C., 1996, "Consider Oxygen for Hydrocarbon Oxidations", Chemical Engineering Progress, 92(11):66-71. 